\title{
Traditional eye medicine practice in Benin-City, Nigeria
}

\author{
JA Ebeigbe
}

Department of Optometry, Faculty of Life Sciences, University of Benin, Nigeria

$<$ jenniferebeigbe@yahoo.com>

Received 11 April 2013; revised version accepted 5 December 2013

\begin{abstract}
The use of traditional eye medicines as a form of eye care in Africa is very common. However, there is concern about the harmful effects of some traditional medicines on the eyes. This study was a cross-sectional survey conducted in Benin-City, Edo state, Nigeria. Sixty-eight traditional medicine practitioners (TMPs) who treated eye conditions participated in this study. Most $(87 \%)$ were males and $13 \%$ were females. Their age ranged between 25 to 65 years with a mean age of $42.25 \pm 2.14$ years. Information was obtained through one-onone oral interviews and a structured questionnaire consisting of open-ended questions. The most common method of training ( $46 \%)$ was by fatherto-son tutelage. Conjunctivitis, itching and poor vision were the most common conditions treated by all practitioners. The majority of the TMPs (62\%), practiced full time while $38 \%$ practiced part time. Forty six percent considered patients' case histories as more important than physical examination of the eye while $54 \%$ felt both history
\end{abstract}

and examination were equally important. Thirtysix percent of practitioners reported inverting the upper eyelids as part of their examination. Couching of cataracts was done by $38 \%$. While $49 \%$ had referred 'difficult' or 'stubborn' cases to orthodox hospitals, $51 \%$ had never referred a case. Traditional healers are well accepted in their communities and will continue to be consulted by the people. Health education programs with emphasis on safe eye care practices need to be established for traditional healers. Working with healers and training them to recognize cases needing urgent referral and encouraging the use of non-harmful practices may provide a more sustainable health care structure in the community. Cooperation between these two aspects of medicine is extremely important for the provision of primary eye care services in rural Africa. ( $S$ Afr Optom 2013 72(4) 167-172)

Key words: Traditional eye medicine, traditional healers, eye diseases, health service utilization, Nigeria

\section{Introduction}

Traditional medicine remains the most widely accessible form of health care in Africa ${ }^{1}$. The traditional medicine programme of the WHO defines traditional medicines as the sum total of all the knowledge and practices whether explicable or inexplicable used in diagnosis, prevention and elimination of physical, mental or social imbalance and relying exclusively on practical experience and preservation handed down from generation to generation whether verbally or written ${ }^{1}$.

In Africa, traditional medicine practitioners (TMPs) or traditional healers are diagnosticians as well as 
prescribers of herbal medicines ${ }^{2,3}$. Rural communities have strong faith in and respect for traditional healers who provide the first line of medical attention for most people in remote areas where modern medical services are inaccessible. Jimba et $\mathrm{al}^{4}$, in their study on the health care seeking behaviour and health policy in rural Nepal, reported that of those who sought health care $81 \%$ first visited traditional healers. While $26 \%$ of them visited traditional healers exclusively, 55\% visited TMPs before seeking care at the health posts.

The services of traditional healers are usually simple, acceptable and affordable to their patients. Evidence supporting the efficacy of traditional treatments remain scanty. While their efficacy has been demonstrated in connection with some medical conditions, harmful effects in some cases have been reported ${ }^{4,5}$. Corneal ulceration is one of the most frequent causes of blindness in developing countries. There are a few published reports of the contribution of traditional eye medicines (TEM) to the development of corneal diseases in Africa, although it is commonly recognised by clinicians to be a significant contributor to corneal ulceration, scarring and blindness ${ }^{10,20,21,24}$. Upadhyay et al ${ }^{6}$ reported that the most common predisposing cause of ulceration was corneal trauma, usually with organic agricultural materials.

Controversies are rift about the place of traditional medicine in modern health care. While some ${ }^{7-9}$ make a case for incorporating it into modern medicine, others are not so well disposed to the fact. In East Africa, $90 \%$ of the population rely on traditional healers. Efforts to synthesize western medicine with traditional healers have been erratic, varying from country to country. In Kenya, traditional healers are held in ill repute by government leaders. Tanzania sponsors a traditional medical research unit, a major laboratory complex for traditional medical research and a chair in traditional medicine at the University of Dar es Salaam. Miller ${ }^{8}$ reported that neither government openly supports traditional medicine for fear of encouraging exploitation, charlatanism and malpractice. The desire on the part of the local medical doctor to disassociate himself from the traditional healers, and be considered totally modern mitigates against synthesis.

In Nigeria, the only form of training of traditional healers is by private individuals paying an agreed sum of money and signing an agreement to go into apprenticeship under a recognized healer. There are no formal plans to incorporate them into the health care system, as most western medical practitioners regard them with suspicion and distrust. There is no inventory of traditional eye medicines nor have investigations been carried out to determine the most commonly used products, those that are particularly harmful and those that might have curative properties.

The last two decades however, have witnessed a worldwide increase in the use of TEMs ${ }^{10-13}$. This is despite that there is little scientific evidence to justify the use of traditional medicines in treating eye diseases. The use of TEMs in Africa is common but research on the subject is difficult because the transfer of knowledge from one healer to another is exclusively verbal $^{14-16}$. Also, different parts of the plant (leaves, bark, roots, et cetera.) are used in different ways ${ }^{17-19}$.

Access to quality health care is said to be a fundamental human right but the numerous challenges faced by the modern health care systems in Nigeria make this a reality only for a small section of the populace. Hence a lot of people, especially those in the rural areas visit TMPs. In Nigeria traditional beliefs differ from one ethnic group to another but the belief in ancestral spirits is one that runs through all the cultures. Some local people believe that some diseases are caused by the spirits or some gods and cannot be cured by western medicine. While some TMPs therefore focus primarily on medicinal plants derived from farms, forest or market, others claim to heal with plants through consultation with ancestral spirits $^{20,21}$.

Understanding the properties of specific traditional eye medicines is complex and the complexity is increased because traditional medicine is dynamic, changing with the cultural, political and economic environment of the settings in which healers live ${ }^{22-24}$. There is also limited information on specific traditional eye practices or traditional eye medicines. The cost of traditional medicine is variable, depending on the nature of the treatment, the kind of disease treated and the relative wealth of the client as perceived by the healer ${ }^{25-27}$.

The World Health Organization ${ }^{1}$ indicated that research on the TMPs themselves along with other areas of their practice be given special attention hence the need for this study, which therefore sought 
to determine the scope of practice of traditional eye medicine practitioners in Benin city, Nigeria as well as ascertain their understanding of common eye ailments.

\section{Method}

This was a cross sectional study, conducted in Benin-City, Edo state. Three local government areas namely Oredo, Egor and Ikpoba-Okha were randomly selected. A total of one hundred (100) traditional healers were recruited for this study. Out of this number, sixty-eight (68) were self proclaimed 'eye doctors' who treated eye conditions as well as other diseases. This made up the study sample. The traditional healers were then interviewed one-on-one with respect to how they arrive at the diagnosis they make and how they treat eye diseases. The study was approved by the University of Benin Research Ethics Committee and informed verbal consent was obtained from the participants before the commencement of the study.

The TMPs were visited in their homes or offices where they usually consulted with their clients. The data collection method involved eliciting information through oral interviews and a structured questionnaire consisting of open-ended questions. The local version of the English language called 'pidgen' was used during the interviews. Traditional healers, who could not read or write, had their questionnaires read out and filled out for them by the interviewer.

Out of the 68 TMPs who participated in this study, $59(87 \%)$ were males while nine $(13 \%)$ were females. Their age range was between 25 to 65 years with a mean age of $42.25 \pm 2.14$ years. Thirty-five $(51 \%)$ of the TMPs, had only primary school education, 33 $(49 \%)$ attended secondary school while none had tertiary education.

\section{Results}

Of the 68 TMPs used in this study, 15 (22\%) had been in practice for 20 to 25 years, 20 (29\%) for 16 to 20 years, $18(26 \%)$ for 11 to 15 years and 15 $(22 \%)$ for less than 10 years. The most common eye conditions treated were conjunctivitis, itching and poor/cloudy vision. These were treated by all $(100 \%)$ the TMPs. This was followed by cataract $(76 \%)$, chalazion $(68 \%)$, corneal ulcer $(53 \%)$, entropion $(35 \%)$ and glaucoma (12\%). Sugar solution was one of the chemical substances used in the treatment of specific eye conditions. Some of the TMPs reported that they had told clients to wash their faces with the first urine passed in the morning on awakening. They considered it as concentrated and potent to cure eye diseases. Instillation of breast milk into the eyes as treatment for red eyes was also a common treatment option.

\section{Mode of training}

The most common method of training (46\%) was by informal apprenticeship such as father-to-son tutelage, learning via a relative (37\%) and formal apprenticeship with payment of money and signed agreement (18\%). The majority of the TMPs (62\%) practice full time and 38\% practice part time having other forms of livelihood. Most of the TMPs reported that the majority of their clients were women and children as most men hardly come for treatment unless their condition is severe. The nine female TMPs interviewed in this study reported that they were also traditional birth attendants.

\section{Method of Examination}

Forty six percent of TMPs considered patients' case histories as more important than the physical examination of the eye, while $54 \%$ felt that both history and examination were equally important. Thirty-six percent of the TMPs reported that in some cases, they inverted the upper eyelids as part of the examination to see if it was 'smooth' or 'rough' but none of them reported using a penlight.

\section{Method of treatment}

All redness of the eye was treated as conjunctivitis, as differentiation of the various forms was too complicated for most of the traditional healers. Epilation of lashes was frequently done by most of the TMPs. Some of the TMPs claimed they 'treated' cataracts by giving various forms of concoction until full maturation after which some of the traditional healers referred their clients to the hospital. Other TMPs performed couching which is a crude attempt at surgery by using force on a fine point object, such as a needle, to knock the lens back into the posterior chamber. 
Above one-third (38\%) of the TMPs performed couching. Forty nine percent referred 'difficult' or 'stubborn' cases to the hospitals and 51\% reported that they had never referred a case. Eight practitioners reported treating glaucoma which they referred to as 'hypertension' of the eye and/or 'high blood pressure' of the eye. Cases of glaucoma were usually of those who had initially visited orthodox hospitals, where the diagnosis was made but felt traditional medicine was more affordable and a faster option for them. Consultation or registration fees are rarely paid. Payment for treatment usually ranges from $\$ 500$ to $\$ 1500$ equivalent of 3 to 10 US dollars per visit.

\section{Need for collaboration?}

On whether they could work with the western trained doctors in promoting eye care, $79 \%$ agreed that it was possible and would be willing to be trained in order to enhance their skills and knowledge. However, $21 \%$ felt that they had sufficient knowledge needed to manage their clients.

\section{Discussion}

In Nigeria, as in most developing countries, modern health care facilities are usually located in the urban areas. As cultures evolve and people migrate to cities or urban areas for jobs or better lifestyles, traditional medicine practice is modified or sometimes abandoned in preference to modern scientific medicine ${ }^{20,21}$. The poor condition of the roads and high cost of transportation pose a huge barrier to the rural dwellers ${ }^{25}$. As a result of this, alternative medicine becomes the first point of call ${ }^{20}$. This accounts for some delay in the utilization of orthodox sources of eye care services. This is an important cause of avoidable blindness, especially in cases where early detection and treatment would have prevented the victim from blindness ${ }^{21}$.

The most common types of traditional eye medicines used by the TMPs were plant extracts, herbs, roots and their derivatives. The efficacy of these roots and herbs is largely unproven and may sometimes actually be toxic to the eye ${ }^{28}$. While some of them may not be more effective than a placebo, the application of others could actually be harmful to the eye. The substances used topically in ocular treatment include naturally occurring plants and extracts, commercial chemicals and fluids from various sources, parts of plants and powder produced from dried plants. These are applied locally to the lids, conjunctiva sac or taken orally. Most of them, especially those with a high $\mathrm{pH}$ or those containing particulate foreign matter, are potentially blinding. Alkaline substances may penetrate and opacify the cornea. Others may damage the corneal epithelium, producing ulceration and even perforation. If microorganisms are introduced in solutions, suppurative keratitis may develop, followed by endopthalmitis ${ }^{20,21}$.

Reports from Nigeria and other parts of Africa have documented the deleterious effects of traditional eye medicines. The adverse effects included worsening of the original condition and predisposition to infections that in some cases totally destroyed the cornea or the eye $\mathrm{e}^{29-31}$. Previous studies ${ }^{30,31}$ have reported adverse consequences of the use of unorthodox medication. TEM use resulting in severe and uncontrolled eye infection had been documented as the indication for surgical removal of the eye ball in $37.5 \%$ of cases in a center ${ }^{31}$. Courtright et al ${ }^{22}$ described the use of traditional eye medicines among patients with corneal diseases in rural Malawi. They found that rural patients commonly consulted traditional healers even after receiving treatment from western trained medical staff.

Some of the traditional healers in this study apart from prescribing and administering roots and herbs also make attempt at eye surgery, in the form of couching. This crude attempt at surgery is a technique of dislodging the lens, thus allowing light to enter the eye. A sharp instrument like a thorn or needle is used to pierce the eye either at the edge of the cornea or sclera near the limbus. The opaque lens is then pushed downwards, allowing light to enter the eye. This would usually give a false improvement in vision because of the dislodged lens. However, couching is a largely unsuccessful technique with abysmal outcomes. Complications of couching include secondary glaucoma, hyphaema and optic atrophy. This study supports Mpyet et al ${ }^{30}$, who reported on couching by traditional healers in Nigeria. Similarly, a recent report of the Nigeria's national blindness and low vision survey revealed that most of the cataract operations in the country were performed by herbalists who couch the affected eyes ${ }^{31}$.

Traditional management of eye diseases is based on the healers' concept of the disease causation as 
well as their knowledge of the herbal, animal and chemical substances that possess (or are reported to possess) remedial effect on the disease. While many of the healers interviewed failed to give a clear distinction between the various eye conditions, diseases such as cataract, which was described as a clouding or whitening of the eye, foreign bodies and injuries were recognized easily by description.

In almost all cases the medicinal substances were first diluted in water before they were applied to the eyes. Traditional healers tend to prefer the use of substances that cause irritation and pain as this is perceived by the healers and their patients as proof of potency. Such substances may be acidic or alkaline resulting in ocular burns. No particular attention is paid to the mode of action (antibiotic or steroid), concentration, and sterility as most of the mixtures are made without regard for hygiene including using contaminated water, local gin, saliva and even urine ${ }^{18-20}$.

Some of the TMPs reported that they referred eye cases that were beyond their capacity to the hospitals. Those who did not refer cases reported that they were not eager to turn their clients to biomedicine as this will be perceived as failure on their part and would affect their financial status. From the interviews conducted, the impression given was that if a client would be referred or not depended on how the TMP perceived himself. If the practitioner believes the ancestors have given him/her the power to cure all conditions, then failure is not an option to be considered and such a practitioner would never refer cases. However, if the practitioner admits that his skills and knowledge are limited to specific conditions, then there is a great possibility that he would refer cases that are beyond his capability to other points of health care. Three factors legitimize the role of the traditional healerstheir own beliefs, the success of their actions and the beliefs of the community.

Moses et $a l^{14}$ in their study on determining the impact of eye care programmes in which TMPs were participants, reported that there was limited success in expanding activities beyond a certain level because referral was low based on marginalization problems but suggested that referral of patients to the hospital should be presented as a collaborative activity with the healer as a vital link in the process whereby sight is preserved or restored. However, Omolase et $a l^{25}$, in a study to find out the perceptions of Nigerian ophthalmologists about traditional eye care practice in Nigeria reported that ophthalmologists were against any form of collaboration with traditional healers.

If a collaborative system were to operate in Nigeria, it might reduce the number of TMPs who do not refer cases to the hospitals. More than half of the TMPs in this study had never referred a case to the hospital. This is a serious cause for concern. If given correct information, some of these traditional healers could form a useful media through which the community can be reached regarding eye care. Contrary to common perception, and provided that they are manufactured in the correct way and administered in the right dosage, some natural medicines could safely promote healing. The WHO notes that "inappropriate use of traditional medicines or practices can have negative or dangerous effects" and that further research is needed to ascertain the efficacy and safety of several of the practices and medicinal plants used by traditional medicine systems ${ }^{12,16}$.

The frequent use of TEMs and the presence of numerous traditional healers in rural areas of Africa suggest an obvious and important need to educate traditional healers on safe eye care practices. Also eye care programmes could benefit greatly by including traditional healers in primary eye care activities.

\section{References}

1. Chan MN. Address at the WHO Congress on Traditional Medicine. World Health Organization 2008866.

2. Mselle J. Visual impact of using traditional medicine on the injured eye in Africa. Acta Tropica 199870 185-192.

3. Foster A, Johnson G. Traditional eye medicines-good or bad news? Br J Ophthalmol 199478 807-810.

4. Jimba M, Poudyal AK, Wakai S. The need for linking healthcare-seeking behaviour and health policy in rural Nepal. Southeast Asian J Trop Med Pub Health 200334 2-3.

5. Chana HS, Schwab L, Foster A. With an eye to good practice: traditional healers in rural communities. World Health Forum 199415 144-146.

6. Upadhyay MP, Karmacharya PC, Koirala S, Tuladhar NR, Bryan LE, Smolin G, Whitcher JP. Epidemiologic characteristics, predisposing factors and etiologic diagnosis of corneal ulceration in Nepal. Am J Ophthalmol 1991111 92-99.

7. Klauss V, Adala HS. Traditional herbal eye medicine in Kenya. World Health Forum 199415 138-43.

8. Miller NN. Traditional medicine in East Africa: the search for a synthesis. Am Univ Field Staff Rep Afr 198022 1-15.

9. Chana HS. Integration of traditional healers into primary 
eye care. J Com Eye Health 199710 3-4.

10. Courtright $\mathrm{P}$, Lewallen S. Traditional healers in prevention of blindness. J Com Eye Health 199710 1-2.

11. Kimani V. The role of traditional medicine in ophthalmology in Kenya. World Health Forum 198317 1827-30.

12. Thylefors B, Negrel AD, Pararajasegaram R, Dadzie KY. Global data on blindness. World Health Org 199573 115121.

13. Poudyal AK, Jumba M, Poudyal BK, Wakai S, Jumba M. Traditional healers' roles on eye care services in Nepal. $\mathrm{Br}$ J Ophthalmol 200589 1250-1253.

14. Moses C, Susan L, Steve K, Harjinder C, Courtright P. Collaboration with African Traditional Healers for the prevention of blindness. Health Forum 200016 158-42.

15. Shenoy R, Bialasiewicz A, Khandekar R, Barwani B, Belushi H. Traditional medicine in Oman: It's role in ophthalmology. Mid East Afr J Ophthalmol 2009 16 92-96.

16. ResnikoffS, Pascolini D, Etya'aleD, KocurI, Pararajasegaram R, Pokharel GP. Global data on visual impairment in the year 2002. World Health Org 200482 844-851.

17. Thylefors B. A global initiative for the elimination of avoidable blindness. Am J Ophthalmol 1998125 90-93.

18. Bisika T, Courtright P, Geneua R, Kasote A, Chimombo L, Chirambo M. Self-treatment for eye diseases in Malawi. Afr J Trad Complem Alt Med 20096 23-29.

19. Ahmad K, Mohammad AK, Mohammad BQ, Mohammad DK, Azam A, Clare G. Use of home remedies and traditional medicines for the treatment of common eye ailments in Pakistan. Pak J Ophthalmol 200925 57-61.

20. Nwosu SNN. Destructive ophthalmic surgical procedures in Onitsha, Nigeria. Niger Postgrad Med J 200512 53-6.

21. Osahon AI. Consequences of traditional eye medication in UBTH Benin-City. Niger J Ophthalmol 19953 51-4.

22. Courtright P, Lewallen S, Kanjoloti S and Divala D J. Traditional eye medicine use among patients with corneal disease in rural Malawi. Br J Ophthalmol 199478 810812.

23. Courtright P. Eye care knowledge and practices among Malawian traditional healers and the development of collaborative blindness prevention programs. Soc Sc Med 199541 1569-1575.

24. Courtright $P$, Lewallen $S$ and Kanjaloti S. Changing patterns of corneal disease and associated vision loss at a rural African hospital following a training programme for traditional healers. Br J Ophthalmol 199680 694-697

25. Omolase CO, Mahmoud AO. Perceptions of Nigerian ophthalmologists about traditional eye care practice in Nigeria. Afr J Med Sci 200837 255-259.

26. Venkatesh P, Pillai M, Manimegalai TK, Srinivasan M. Use of traditional eye medicines by corneal ulcer patients presenting to a hospital in South India. Ind J Ophthalmol 199947 15-18.

27. Courtright $\mathrm{P}$, Chirambo M. Symposium report: Traditional healers. J Com Eye Health 199710 6-7.

28. Shimobiraki C, Jimba M. Traditional vs modern medicine: which healthcare option do rural Nepalese see? Technol Developm 200215 47-55.
29. Nyenze E, Ilako O, Karimurio J. Knowledge, attitude and perception of traditional healers on treatment of eye diseases in Kitui district of Kenya. East Afr J Ophthalmol $2007 \mathbf{1 3}$ 6-11.

30. Mpyet C, Direen B, Solomon AW. Couching and orthodox cataract surgery in Nigeria. Br J Ophthalmol 2005189 936938.

31. Eze BI, Chuka-Okosa C, Uche J N. Traditional eye medicine use by newly presenting ophthalmic patients to a teaching hospital in south-eastern Nigeria: Socio-demographic and clinical correlates. BMC Complement Alt Med 2009 9 40-44. 